Original Research Paper

\title{
Early Detection of Alzheimer's Disease using Convolutional Neural Network Architecture
}

\section{Deepthi Kamath $^{1}$, Misba Firdose Fathima ${ }^{1}$, Monica K. P ${ }^{1}$, Kusuma Mohanchandra ${ }^{1}$ \\ ${ }^{1}$ Department of Information Science and Engineering, Dayananda Sagar Academy of Technology and Management, Bangalore. Karnataka, India.}

Article History

Received:

23.06.2021

Revised:

27.08.2021

Accepted:

02.09 .2021

*Corresponding Author:

Deepthi Kamath

Email

misbafirdose66@gmail.com

This is an open access article, licensed under: $\mathrm{CC}-\mathrm{BY}-\mathrm{SA}$

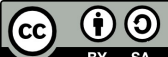

Abstract: Alzheimer's disease is an extremely popular cause of dementia which leads to memory loss, problem-solving and other thinking abilities that are severe enough to interfere with daily life. Detection of Alzheimer's at a prior stage is crucial as it can prevent significant damage to the patient's brain. In this paper, a method to detect Alzheimer's Disease from Brain MRI images is proposed. The proposed approach extracts shape features and texture of the Hippocampus region from the MRI scans and a Neural Network is used as a Multi-Class Classifier for detection of AD. The proposed approach is implemented and it gives better accuracy as compared to conventional approaches. In this paper, Convolutional Neural Network is the Neural Network approach used for the detection of AD at a prodromal stage.

Keywords: Alzheimer's Disease, Convolutional Neural Network, Magnetic Resonance Imaging. 


\section{Introduction}

Alzheimer's disease (AD) is an irreversible, accelerative neurological brain disorder marked by a decline in cognitive functioning with no validated disease altering the treatment. AD is the most regular type of dementia, and is one of the major challenges for healthcare in the twenty-first century [1].

Overall, a great deal of endeavour has been made in order to develop strategies for early detection of $\mathrm{AD}$, especially at pre-symptomatic stages in order to slow down or prevent disease. In particular, advanced neuroimaging techniques, such as magnetic resonance imaging (MRI) have been developed and used to identify AD-related structural and molecular biomarkers [2] [3] [4].

The cortex in the brain patient shrinks and this shrinkage occurs particularly in the hippocampus region.This region is involved in all of the capabilities that is related to thinking, reasoning and making new memories.Brain ventricles, which produce cerebrospinal fluid, also become larger in an $\mathrm{AD}$ patient [5] [6].

In the prodromal stage of Alzheimer's, individuals may function independently. Regardless of this, the person might feel that he or she is having memory lapses, for example forgetting familiar words or location of everyday objects [7] [8].

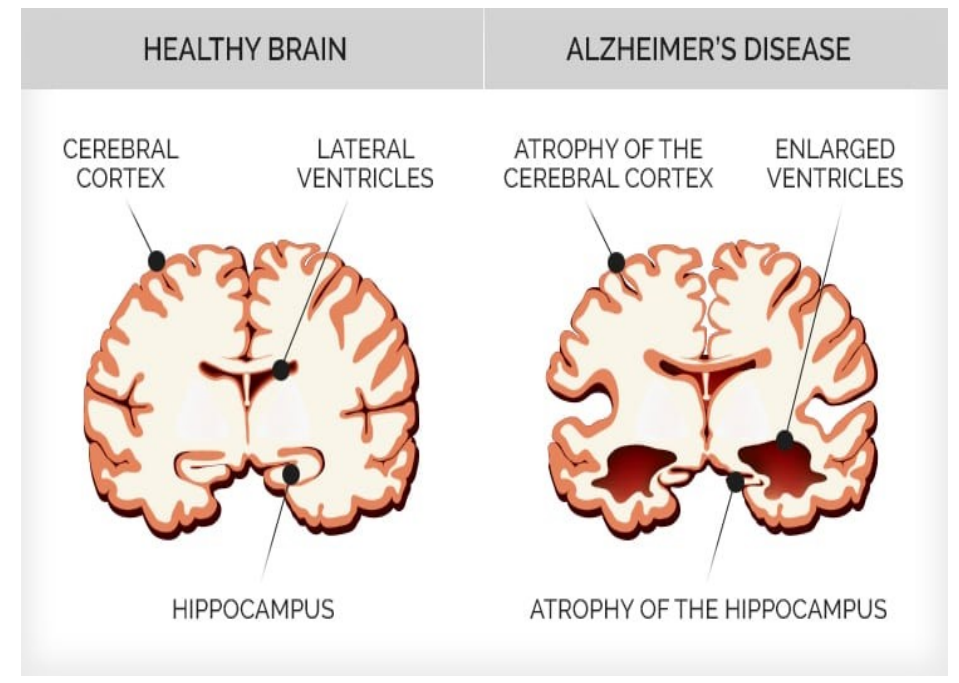

Figure 1. Comparison of Normal VS Affected Alzheimer's Brain [7]

Some recent studies reveal that $\mathrm{AD}$ may begin 20 years or more before any symptoms appear and the disease is clinically diagnosed. Only after a certain stage, patients may experience diagnostic symptoms such as deterioration in memory and decline in cognitive abilities when the irreversible neurological damage already occurs [8] [9].

Therefore, early and accurate diagnosis of $\mathrm{AD}$ is crucial and may be possible via computer-assisted analytical techniques. Receiving an early diagnosis of $\mathrm{AD}$ will enable patients to benefit from various treatments, plan their future, and maximize their life quality [10].

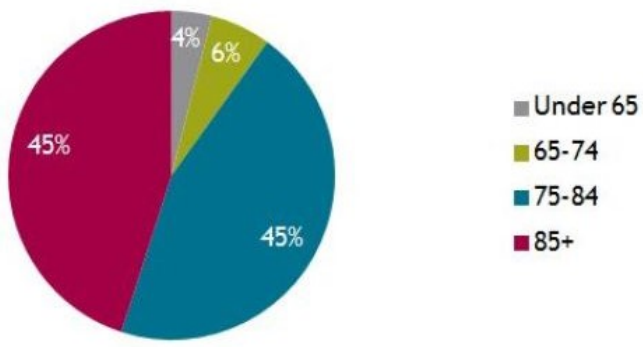

Figure 2. Patients Affected by Alzheimer's Disease Considering AGE as a Factor [10] 
As per the reports, the below pie chart gives us a clear picture of the people who are mostly affected by $\mathrm{AD}$ considering age as an element shown in Figure 2.

\section{Convolutional Neural Network (CNN)}

Convolutional neural network is a deep Feed-forward Neural Network (FNN) that comprises multilayers of artificial neurons, which provides good performance in large-scale segmentation, processing of image, classification and also for other auto correlated data.

The foundation for CNNs are filters i.e. kernels. Kernels use the convolution operation in order to fetch all the relevant features from the input. The importance of filters using images as input data is as shown in the Figure 3 [11].

CNN was initially introduced to solve problems related to image data. But it has been observed that they perform significantly well on sequential inputs as well [12] [13].

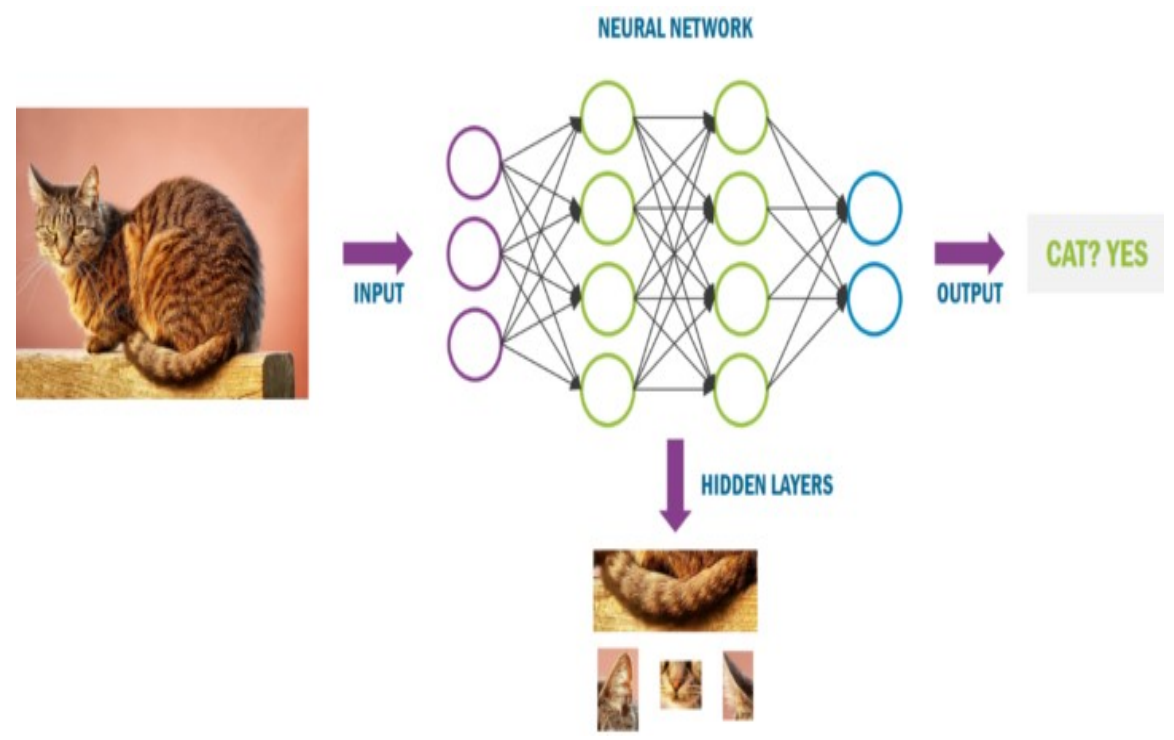

Figure 3. Convolutional Neural Network - Image Classification [11]

Advantages of $C N N[14]$ :

- CNN automatically learns about the filters without referring to it explicitly. They come in handy during the extraction of relevant features from the input data.

- $\mathrm{CNN}$ fetches the spatial features of an image. Spatial features refer to the pixel arrangement and the relationship between the pixels in an image. They aid us in determining the object accurately, the location of an object, as well as its relationship existing between other objects in an image.

CNNs have ample applications in image, video recognition, recommender systems and natural language processing.For an instance let us consider related to Computer Vision. However, the basic concept remains the same and can be applied to any other use-case [15].

In a typical CNN layer, we make a choice to either have a stack of $3 \times 3$ filters, or a stack of $5 \times 5$ filters or a max pooling layer. In general all of these are beneficial to the modelling power of the network. The inception module suggests the use of all of them.

This means instead of adding a particular filter size layer, we add all $1 \times 1,3 \times 3,5 \times 5$ filters and perform convolution on the output from the previous layers. Since pooling has been essential for the success of current CNNs, the inception module also includes an additional pooling path. The output of all the filters are concatenated and passed on as input to the next layer [16]. 


\section{Methodology}

In this section, the main components of our framework are presented. In the proposed approach, various shape and texture features are extracted from the hippocampus for detection of Alzheimer's disease. From the training dataset features are extracted using Convolution Neural Network (CNN) an advanced deep learning method and a training model is processed and it gives a validation model.

Test data has to be given to the validation model which was built using $\mathrm{CNN}$, the validation model has to extract the features from the input MRI scan image and classify the Alzheimer category [17] [18].

\subsection{Datasets and Tools}

In this research, OASIS Dataset used.

\section{OASIS}

The Open Access Series of Imaging Studies (OASIS) is a research aimed at making magnetic resonance imaging (MRI) data sets of the brain freely available to the scientific community.

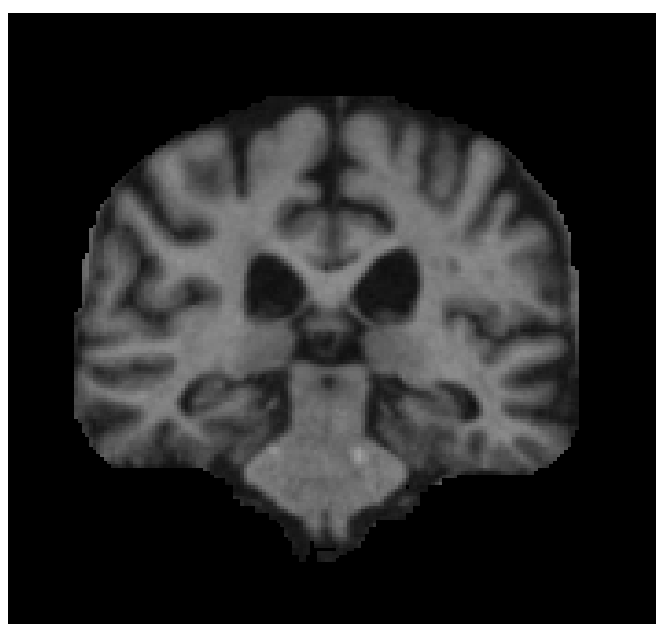

Figure 4. Sample-1 Alzheimer's affected Brain MRI

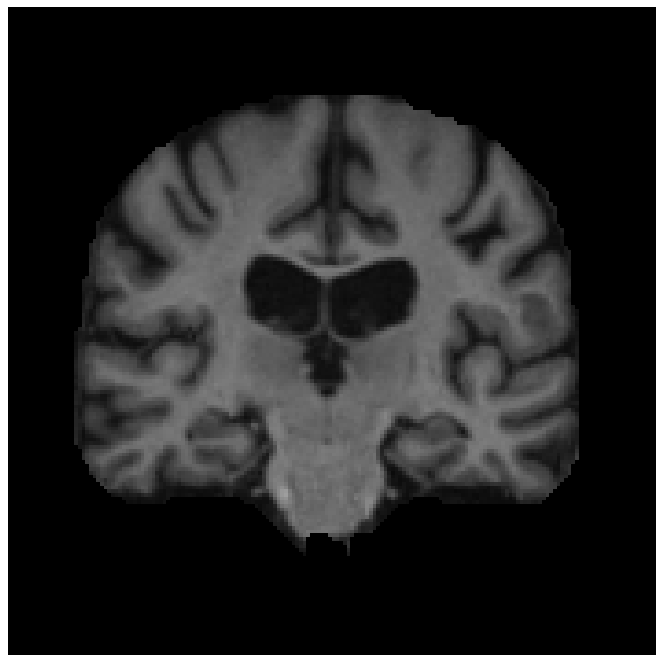

Figure 5. Sample-2 Alzheimer's affected Brain MRI 


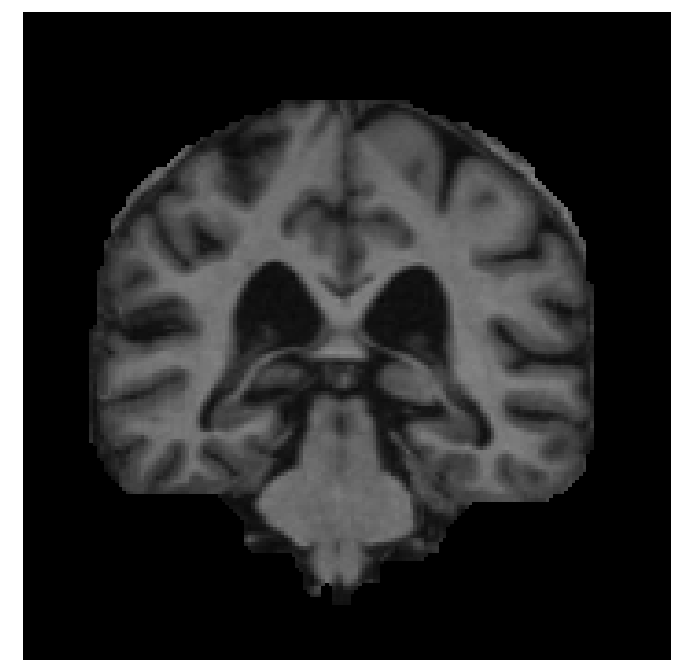

Figure 6. Sample-1 Normal Brain MRI Image

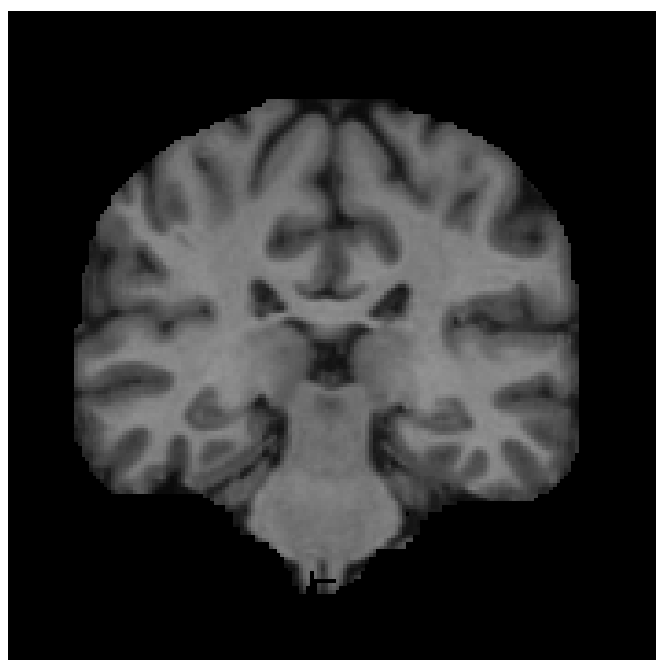

Figure 7. Sample-2 Normal Brain MRI Image

Therefore with the facility of making the dataset available this can help in the near future discoveries related to neuroscience.

Precisely, the OASIS dataset is intended to play multiple roles. Initially, OASIS images and associated computations serve as data sets for continued scientific exploration.

\section{Keras}

Keras an API which is fundamentally designed for human beings. It observes best practices for reducing cognitive load, offers consistent \& simple APIs, minimizes the number of user actions required for common use cases, and it provides clear \& actionable error notifications. It also has huge documentation and developer guides. 


\section{TensorFlow}

TensorFlow is an open source software incorporated in machine learning. It has an extensive, flexible ecosystem of tools, libraries and community resources that allows researchers to push the state-of-theart in ML and the developers can conveniently build and deploy ML powered applications.

\section{OpenCV}

OpenCV (Open Source Computer Vision Library) is a huge open source library which is used for computer vision, image processing and machine learning. OpenCV has an ability to provide a familiar infrastructure for computer vision applications and to stimulate the use of machine perception in the commercial products.

\section{Confusion Matrix}

Confusion Matrix is an approach which briefs the performance of a classification algorithm. Classification accuracy solely can be spurious if there are an unequal number of observations in each class or if there are more than two classes in the dataset.

Overall confusion matrix can give you a clear picture of what the classification model is getting right and what type of errors it is making.

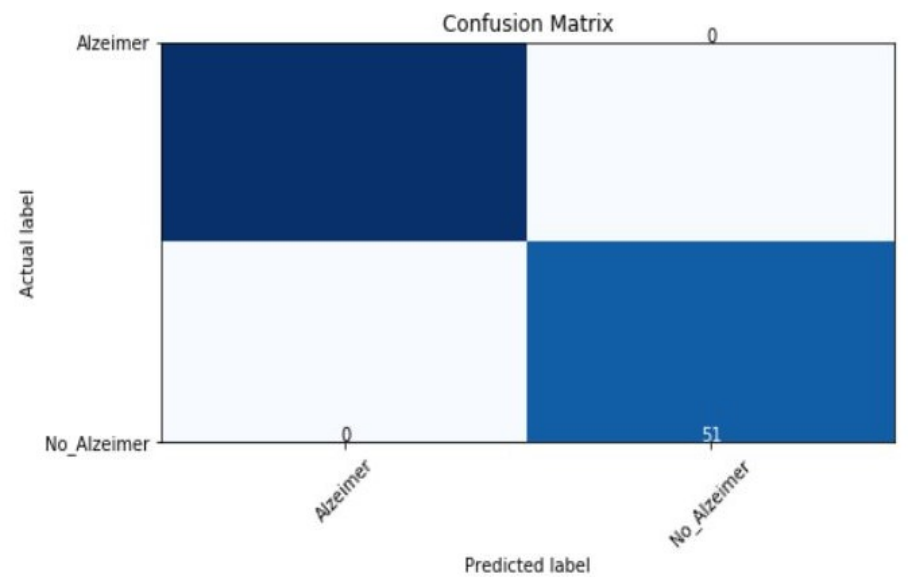

Figure 8. Confusion Matrix for Test Model

Figrue 8 is a confusion matrix for the test dataset of 113 images which classifies 62 Alzheimer's datasets as Alzheimer's and 51 Non Alzheimer's dataset to No_Alzheimer's accurately.

\subsection{Data pre-processing}

Data preprocessing is a mechanism for assembling and processing the raw data and making it acceptable for the machine learning model. It is an initial and a significant step while creating a machine learning model.

In this module the data which we have retrieved from OASIS is further resized for future use. Image resizing, or image scaling, is a geometric image transformation which modifies the image size based on an image interpolation algorithm. This image gauging process can enlarge or reduce the resolution of a target image dataset so that the absolute size of the image dataset is altered.

Computers are able to perform computations on numbers and are unable to interpret images in the way that we do. Further we have to convert the image dataset to numerical format for the computer to understand. The image will be converted to grayscale (range of gray shades from white to black) the computer will assign each pixel a value based on how dark it is. The numerical format is put into an array and the computer does computations on that particular array. We then feed the resulting array for the next step. 
It is important that we come across clean and formatted data. So for this, we use data preprocessing tasks. In this step we have retrieved the data from the OASIS dataset and this dataset should be in a specific format of jpeg. We have converted all the images to the same shape i.e, all the images to the same base resolution. This dataset is then further put in a trained model that can take upto millions of images and detects whether the person is suffering from Alzheimer's or not.

\subsection{Model Construction}

We are building our model by using a Convolutional Neural Network (CNN) which is a special architecture of artificial neural networks.

$\mathrm{CNN}$ efficiently reduces the number of model parameters by inserting convolution and pooling layers that lead to a reduction in complexity.

CNN uses some features of the visual cortex. Now that we're done with pre-processing, we can start implementing our neural networkAs a next step we will have 3 convolution layers with 2 x 2 maxpooling.

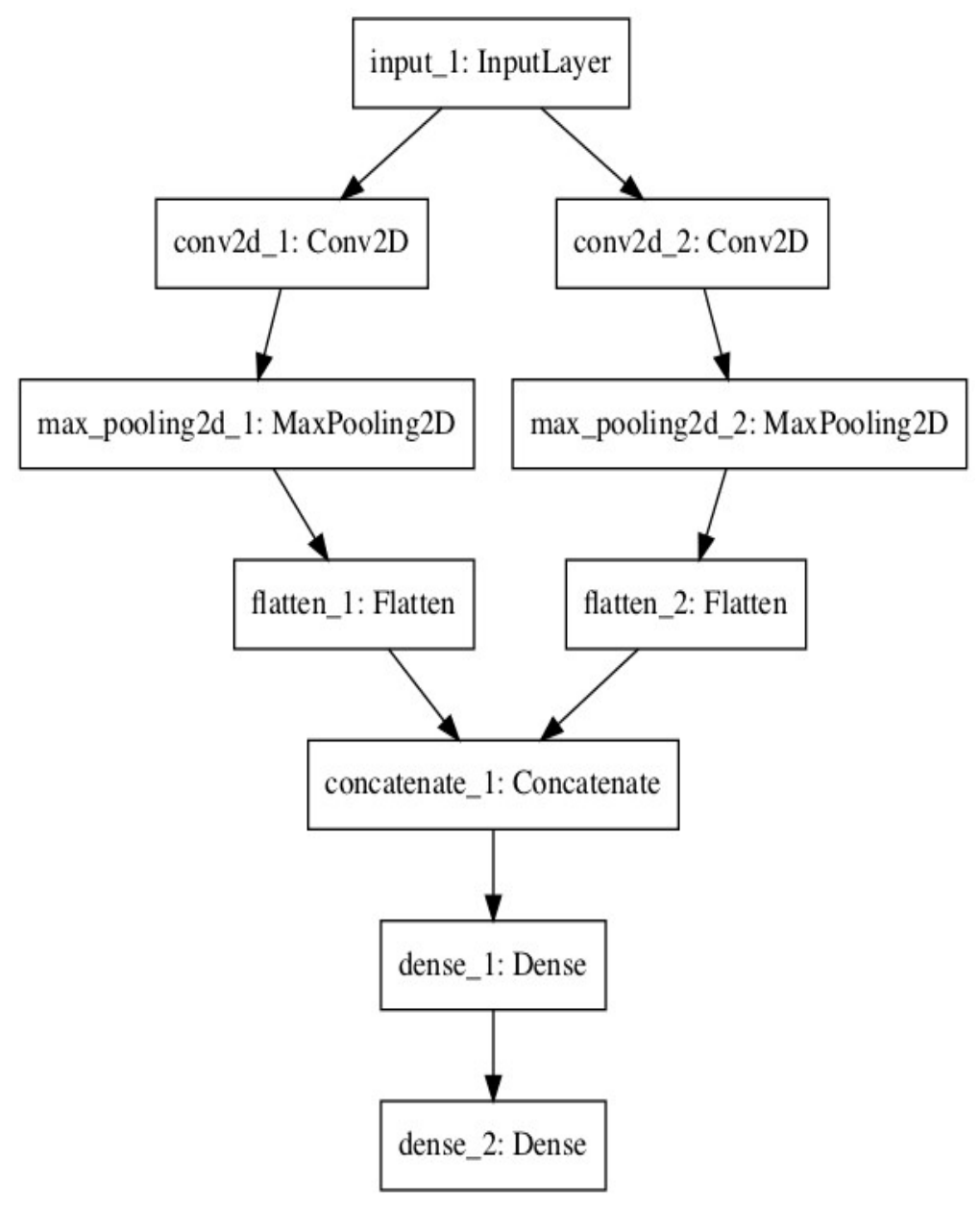

Figure 9. Architecture of our CNN Sequential model

The Sequential model API is pronounced for developing deep learning models in most conditions (A CNN model). 
The below figure shows The Sequential model API which is a way of creating deep learning models where an instance of the Sequential class is created and model layers are created and added.

A Sequential model is pertinent for a plain stack of layers where each layer has exactly one input tensor and one output tensor.The common architecture of ConvNets is a sequential architecture.

Max-Pooling is a mechanism used to reduce the dimensions of an image by taking the maximum pixel value of a grid. This helps in reducing overfitting and makes the model more generic. After that, we add 2 fully connected layers. Since the input of fully connected layers should be two dimensional, and the output of the convolution layer is four dimensional, we need a flattening layer between them. Softmax layer is present at the very end of a fully connected layer.

The Dropout layer irregularly sets input units to 0 with a frequency of rate at each step during training time, which intercept overfitting.

\subsection{Training and Testing the CNN Model}

In the datasets we collected, we have split $80 \%$ of the data as training data, and the rest $20 \%$ of the data as test data.

After model construction is done it is time for model training. We were able to build an artificial convolutional neural network that can recognize images. Here we split the dataset into train and test dataset as mentioned above. Finally we will build and train the model using the training dataset.

Once the model has been trained by feeding the data it is now possible to carry out the model for testing. In the course of this phase a test set of data is being loaded.This data set has never been fed to the model and therefore it's true accuracy will be verified. Eventually, the saved model can be used in the real world. The name of this phase is model evaluation. Thus the model is now in a position to evaluate the new data.

\section{Result}

The proposed system produces the output stating if there is Alzheimer's or not with the overall accuracy of above $95 \%$.

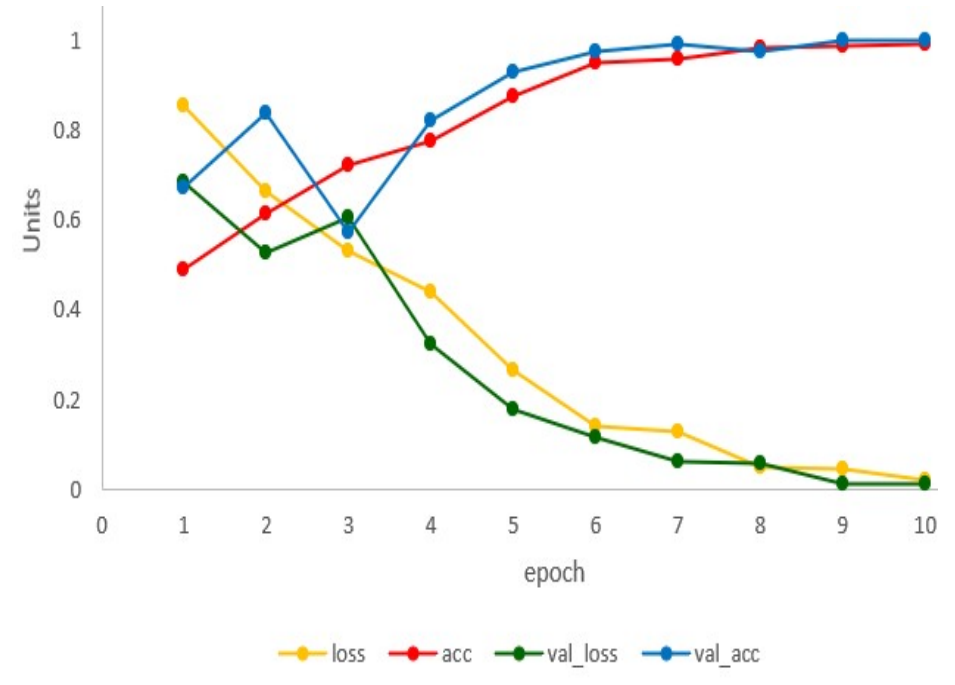

Figure 10. Values of loss, accuracy, val_loss and val_accuracy

Figure 10 represents the values of loss, accuracy, val_loss and val_accuracy with respect to epoch.

\section{Conclusion}

In this study, we proposed a novel method for the early detection of AD using CNN. CNN is more suitable for image processing especially in image classification. This model is used to predict Alzheimer's Disease affected-brain v/s a normal aging brain and is able to do so with higher accuracy 
by using brain MRI data. We have retrieved the data from the OASIS dataset. Henceforth we can conclude that the proposed system in this pape gives an overall accuracy of more than $95 \%$ using CNN.

\section{Acknowledgement}

We express our sincere gratitude to our guide Dr. M. Kusuma for her constant help, encouragement and motivation throughout the research. Data collection and sharing for this research was provided by OASIS.

\section{References}

[1] G. Harshitha, C. Chamarajan, and Y. Charishma, "Alzheimer's Disease: A Survey", International Journal of Artificial Intelligence, vol. 8, no. 1, pp. 33-39, Jun. 2021.

[2] S. Saman, and G. Tofighi, "Alzheimer's Disease Neuroimaging Initiative - DeepAD: Alzheimer's disease classification via dkr7eeep convolutional neural networks using MRI and fMRI," Journal of BioRxiv, vol. 7, no. 441, 2016.

[3] S. Saman, and G. Tofighi, "Classification of Alzheimer's disease structural MRI data by deep learning convolutional neural networks," arXiv preprint arXiv:1607.06583, 2016.

[4] R. Jain, N. Jain, A. Aggarwal, and D. J. Hemanth, D. J, "Convolutional neural network based Alzheimer's disease classification from magnetic resonance brain images," Cognitive Systems Research, vol. 57, pp. 147-159, 2019.

[5] I. Jyoti, and Y. Zhang, "Brain MRI analysis for Alzheimer's disease diagnosis using an ensemble system of deep convolutional neural networks," Brain informatics, vol. 5, no. 2, 2018.

[6] D. Kamath, M. F. Fathima, M. K. P., and M. Kusuma, "Survey on Early Detection of Alzheimer's Disease using Different Types of Neural Network Architecture", International Journal of Artificial Intelligence, vol. 8, no. 1, pp. 25-32, Jun. 2021.

[7] K. Oh, Y. C. Chung, K. W. Kim, W. S. Kim, and I. S. Oh, I. , "Classification and visualization of Alzheimer's disease using volumetric convolutional neural network and transfer learning," Scientific Reports, vol. 9, no. 1, pp. 1-16, 2019.

[8] F. Al-azdi, R. Passarella, A. Susanto, C. Caroline, R. D. Puspa, and T. W. Yudha, "Design of A Convolutional Neural Network System to Increase Diagnostic Efficiency of Alzheimer's Disease," in IOP Conference Series: Materials Science and Engineering, vol. 648, no. 1, pp. 12-18, Oct 2019.

[9] A. Farooq, S. Anwar, M Awais, and S. Rehman, "A deep CNN based multi-class classification of Alzheimer's disease using MRI," in 2017 IEEE International Conference on Imaging Systems and Techniques, pp. 1-6, Oct 2017.

[10] S. Liu, C. Yadav, C. Fernandez-Granda, and N. Razavian, "On the design of convolutional neural networks for automatic detection of Alzheimer's disease," Machine Learning for Health Workshop PMLR, pp. 184-201, April 2020.

[11] D. Kamath, M. F. Fathima, K. P. Monica, and M. Kusuma, "Survey on Early Detection of Alzheimer's Disease using Different Types of Neural Network Architecture," IJARIIE, pp. 2395-4396, 2020.

[12] I. Jyoti, and Y. Zhang, "Ensemble of deep convolutional neural networks for Alzheimer's disease detection and classification," arXiv preprint arXiv: 1712.01675, 2017.

[13] A. Mehmood, M. Maqsood, M. Bashir, and Y. Shuyuan, "A Deep Siamese Convolution Neural Network for Multi-Class Classification of Alzheimer Disease," Brain Sciences, vol. 10, no. 2, pp. 84-91, 2020.

[14] K. Alexander, K. Aderghal, A. Krylov, G. Catheline, and J. Benois-Pineau, "3D Inceptionbased CNN with sMRI and MD-DTI data fusion for Alzheimer's Disease diagnostics," arXiv preprint arXiv:1809.03972, 2018.

[15 W. Michael, "Interpretable 2D and 3D Convolutional Neural Networks for Alzheimer's Disease in Brain Scans," arXiv preprint arXiv:1809.04912, 2021.

[16] Y. Huang, J. Xu, Y. Zhou, T. Tong, X. Zhuang, "Alzheimer's Disease Neuroimaging Initiative (ADNI): Diagnosis of Alzheimer's Disease via Multi-Modality 3D Convolutional Neural Network," Frontiers in Neuroscience, vol. 13, pp. 509-516, 2019. 
[17] J. Taeho, K. Nho, and A. J. Saykin, "Deep learning in Alzheimer's disease: diagnostic classification and prognostic prediction using neuroimaging data," Frontiers in Aging Neuroscience, vol 11, pp. 220-229, 2019.

[18] E. Yagis, L. Citi, S. Diciotti, C. Merzi, W. Atnafu, and G. S. De Herrera, "3D Convolutional Neural Networks for Diagnosis of Alzheimer's Disease via structural MRI," arXiv preprint arXiv:1809.09992, 2020. 